\title{
Inter-laboratory survey of erythrocyte free protoporphyrin quantification - announcement of a pilot study
}

\author{
Michael Vogeser ${ }^{1, *}$, Wilhelm Müller ${ }^{2}$ and \\ Thomas Stauch ${ }^{3}$ \\ ${ }^{1}$ Institute of Clinical Chemistry, University of \\ Munich, Munich, Germany \\ ${ }^{2}$ Chromsystems, Munich, Germany \\ ${ }^{3}$ Labor Professor Seelig \& Kollegen, Karlsruhe, \\ Germany
}

Keywords: Erythropoietic protoporphyria (EPP); interlaboratory survey.

Erythropoietic protoporphyria (EPP; E80.0, ICD-10) is an inherited disorder resulting from partially deficient activity of ferrochelatase, the last enzyme in the heme biosynthetic pathway (1). In EPP, protoporphyrin accumulates in erythrocytes, bile, and skin, with photoactivation of accumulated protoporphyrin causing endothelial injury. Thus, the main symptom of EPP is painful cutaneous photosensitivity. Diffuse edema of the skin in sun-exposed areas may resemble angioneurotic edema; in most cases, however, burning and itching initially occur without obvious skin damage. Typically the disease becomes symptomatic before the age of 2 years, but diagnosis is typically delayed by 10 years (2), and late-onset cases have been recognized in hematological diseases (3). Severe and in some cases fulminating liver disease occurs in $5 \%$ of EPP patients, potentially requiring liver transplantation $(4,5)$. The disease has an autosomal pseudodominant, and in some cases autosomal recessive pattern of inheritance $(6,7)$, and a variety of mutations have been found to cause EPP (8). The disorder has only been clearly recognized since 1961 (9). Exact data on the prevalence of EPP are not available: in the United Kingdom 389 EPP cases were documented in 2006 (2), but substantially more cases must be assumed.

An increased concentration of free protoporphyrin in erythrocytes is the laboratory hallmark of EPP. "Free" protoporphyrin (protoporphyrin without any central atom) is indeed the only heme pathway intermediate that accumulates significantly in this disease. Although a number of conditions such as iron-deficient erythropoiesis, lead intoxication, and hemato-

*Corresponding author: Michael Vogeser, MD, Institute of Clinical Chemistry, Hospital of the University of Munich,

Marchioninistraße 15, 81377 Munich, Germany

Phone: +49-89-70953221, Fax: + 49-89-70956220,

E-mail: michael.vogeser@med.uni-muenchen.de logical diseases can lead to increased erythrocyte zinc protoporphyrin concentrations, significantly increased free protoporphyrin concentrations are assumed to be found exclusively in EPP. Slightly increased levels of free protoporphyrin are observed under conditions of zinc depletion and iron deficiency, sometimes in sideroblastic anemia and other hematological diseases, and in homozygous or compound heterozygous porphyrias such as hepatoerythropoietic porphyria, Doss porphyria ( $\delta$-aminolevulinate dehydratase-deficiency porphyria) and congenital erythropoietic porphyria (Morbus Guenther). However, clinically relevant concentrations are never attained in these cases.

Since EPP does not present with visible or characteristic skin abnormalities in many cases (10), erythrocyte protoporphyrin measurement is of essential importance for primary diagnosis of EPP in children and adolescents with avoidance behavior towards sun exposure. In the follow-up of EPP patients, erythrocyte protoporphyrin measurement can potentially help (in addition to quantification of fecal protoporphyrin excretion and the isomeric ratio of coproporphyrin in urine) in early recognition of transition of the disease to a fulminate phase, in which worsening cholestasis leads to further accumulation of protoporphyrin in a vicious cycle $(4,5)$.

Quantification of free erythrocyte protoporphyrin concentrations still represents an analytical challenge and is performed in only a few specialized laboratories. The first approach to laboratory diagnosis of EPP was the demonstration of fluorescent red cells ("fluorocytes") in blood films viewed under ultraviolet light (11). Several chromatographic methods for specific quantification of erythrocyte free protoporphyrin were subsequently described $(12,13)$. In such assays, sample preparation with full-recovery analyte extraction is delicate and the chromatographic separation of free from zinc-chelated protoporphyrin is demanding.

Blood-based materials for calibration and quality control of erythrocyte free protoporphyrin quantification are not commercially available at present and no proficiency testing schemes have been implemented. Indeed, there are no data published on agreement between free erythrocyte measurements performed in different laboratories. We therefore decided to organize an inter-laboratory survey study of free erythrocyte protoporphyrin measurement. Data from this survey will be important in achieving harmonization of protoporphyrin measurement in the future.

For this survey, $30 \mathrm{~mL}$ of heparinized whole blood was sampled from an EPP patient after informed con- 
sent and approval by the Institutional Review Board. Erythrocytes were separated from the plasma by centrifugation, and washed with saline. Aliquots of the sample were prepared and lyophilized in lightprotected vials in an industrial setting (Chromsystems, Munich, Germany).

We can now invite interested colleagues to request an aliquot of this sample (michael.vogeser@med.unimuenchen.de) for free erythrocyte protoporphyrin measurement at no cost. The resulting data will be evaluated after anonymization. It is intended to create a research group with the participants of this survey and to submit the data from this pilot investigation for potential publication in Clinical Chemistry and Laboratory Medicine.

\section{Acknowledgements}

This study is supported by the Hans-Fischer-Gesellschaft, Munich, Germany

\section{References}

1. Anderson KE, Sassa S, Bishop DF, Desnick RJ. Disorders of heme biosynthesis: $\mathrm{X}$-linked sideroblastic anemia and the porphyrias. In: Scriver CR, Beaudet AL, Sly WS, Valle $D$, eds. The metabolic and molecular bases of inherited disease, 8th ed. New York: McGraw-Hill, 2001:29913062.

2. Holme SA, Anstey AV, Finlay AY, Elder GH, Badminton MN. Erythropoietic protoporphyria in the UK: clinical features and effect on quality of life. $\mathrm{Br} J$ Dermatol 2006;155:574-81.
3. Bharati A, Badminton MN, Whatley SD, O'Brien DV, Bell HK. Late-onset erythropoietic protoporphyria in association with haematological malignancy. Clin Exp Dermatol 2006;31:668-70.

4. Doss MO, Frank M. Hepatobiliary implications and complications in protoporphyria, a 20-year study. Clin Biochem 1989;22:223-9.

5. Gross U, Frank M, Doss MO. Hepatic complications of erythropoietic protoporphyria. Photoderm Photoimmun Photomed 1998;14:52-7.

6. Cox TM. Erythropoietic protoporphyria. J Inher Metab Dis 1997;20:258-69.

7. Puy H, Deybach JC, Gouya L, Robreau AM, Bourgois M, Lamoril J, et al. The penetrance of dominant erythropoietic porphyria is modulated by expression of wildtype FECH. Nat Genet 2002;30:27-8.

8. Sassa S. Modern diagnosis and management of the porphyrias. Br J Haematol 2006;135:281-92.

9. Magnus IA, Jarrett A, Prankerd TA, Rimington C. Erythropoietic protoporphyria. A new porphyria syndrome with solar urticaria due to protoporphyrinaemia. Lancet 1961;278:448-51.

10. Lecluse AL, Kuck-Koot VC, van Weelden H, Sigurdsson V, Russel IM, Frank J, et al. Erythropoietic protoporphyria without skin symptoms - you do not always see what they feel. Eur J Pediatr 2008;167:703-6.

11. Rimmington C, Cripps DJ. Biochemical and fluorescence microscopy screening test for erythropoietic protoporphyria. Lancet 1965;1:624-6.

12. Ho J, Guthrie R, Tieckelmann H. Quantitative determination of porphyrins, their precursors and zinc protoporphyrin in whole blood and dried blood by highperformance liquid chromatography with fluorimetric detection. J Chromatogr 1987;417:269-76.

13. Sato $H$, Ido K, Kimura K. Simultaneous separation and quantification of free and metal-chelated protoporphyrins in blood by three-dimensional HPLC. Clin Chem 1994;40:1239-44. 